\title{
BMC Surgery reviewer acknowledgement, 2014
}

Guangde Tu

\section{Reviewer acknowledgement}

The editors of BMC Surgery would like to thank all our reviewers who have contributed their time to the journal in Volume 14 (2014).

\section{Contributing reviewers}

Tamer Abdelbaki

Egypt

Thad Abrams

USA

Walid Abu Arab

Canada

Tomohiro Adachi

Japan

Mohsen Adib-Hajbaghery

Iran

Sunil Agarwal

India

Rajesh Aggarwal

United Kingdom

Magnus Ågren

Denmark

Ferdinando Agresta

Italy

Annette B. Ahrberg

Germany

Clemens Aigner

Austria

Sami Akbulut

Turkey

Melih Akinci

Turkey
Luca Antonio Aldrighetti

Italy

Marco Allaix

Italy

William Allum

United Kingdom

Emad Aly

United Kingdom

Roland Andersson

Sweden

Martin Angele

Germany

Stavros Antoniou

Greece

Saif Anwaruddin

USA

Theerachai Apivatthakakul

Thailand

Asif Ayaz

India

Egemen Ayhan

Turkey

Nather Aziz

Singapore

Marcus Bahra

Germany
Seung Hyuk Baik

South Korea

Gian Luca Baiocchi

Italy

Andreas Bakoyiannis

Greece

Carlo Banfi

Switzerland

Gordon Bannister

United Kingdom

Nikolaos Barbetakis

Greece

Louise Barbier

France

Irene Bargellini

Italy

Neal Barshes

USA

Giacomo Batignani

Italy

Hasan Batirel

Turkey

Dirk Bausch

Germany

Jens Bedke

Germany

Correspondence: guangde.tu@biomedcentral.com

BioMed Central, Room 1006-1007 Financial Plaza, No. 333 Jiujiang Road,

Huangpu District 200001 Shanghai, China 
Marcelo Beltran

Chile

Christophe Berney

Australia

Frederik Berrevoet

Belgium

Samer Bessa

Egypt

Ashish Bhalla

India

Deepraj Bhandarkar

India

Roland Biber

Germany

Franck Billmann

Germany

Marcel Binnebosel

Germany

Desmond Birkett

USA

Alessandro Boellis

Italy

Marja Boermeester

Netherlands

Stefano Bogliolo

Italy

Therezia Bokor

Germany

Patrick Boland

USA

Giuseppe Borzellino

Italy

Sotirios Botaitis

Greece

Sergiu Botolin

USA

Mahdi Bouassida

Tunisia

Michael Brauckhoff

Norway

Marius Bredell

Switzerland

Stefan Breitenstein

Switzerland
Brandon Broome

USA

Merribeth Bruntz

USA

Jacek Budzynski

Poland

Orhan Bulut

Denmark

Jakob Burcharth

Denmark

Neslihan Cabioglu

Turkey

Michael Calderwood

USA

Fabio Campanile

Italy

Giampiero Capobianco

Italy

Fernando Carbonell-Tatay

Spain

Cédric Carrié

France

Ross Carruthers

United Kingdom

Lucas Castro Alves

Brazil

Juan Cata

USA

Fausto Catena

Italy

Abigail Caudle

USA

Marco Ceresoli

Italy

Hakan Ceyran

Turkey

Nohra Chalouhi

USA

Shi-Min Chang

China

Grigoris Chatzimavroudis

Greece

Gar-Yang Chau

Taiwan
Yen-Lin Chen

Taiwan

Michael Chen

Taiwan

Wei Chen

China

Yong-Long Chi

China

Jy Ming Chiang

Taiwan

Sonja Chiappetta

Germany

Vitoon Chinswangwatanakul

Thailand

Charing Chong

Hong Kong

Giovanni Conzo

Italy

Riccardo Corbetta

Italy

Andrea Saverio Corriero

Italy

Wilson Costa

Brazil

Marie Crandall

USA

Derly Cuellar

USA

Steven Cunningham

USA

Adrien Daigeler

Germany

Piergiorgio Danelli

Italy

Marcioi De Moraes

Brazil

Belinda De Simone

Italy

Faramarz Dehghani

Germany

Ismail Demiryilmaz

Turkey

Timm Denecke

Germany 
Jacopo Desiderio

Italy

Andrew Deynichenko

Ukraine

Salomone Di Saverio

Italy

Gaetano Di Vita

Italy

Marius Distler

Germany

Cornelia Dotzenrath

Germany

Michael Douek

United Kingdom

Jianjun Du

China

Xavier Ducrocq

France

Ender Dulundu

Turkey

Traian Dumitrascu

Romania

Gülsüm Özlem Elpek

Turkey

Tobias Else

Germany

Xiaoen Hua

China

Itaru Endo

Japan

Giorgio Ercolani

Italy

Mert Erkan

Germany

Unal Erkorkmaz

Turkey

Eloy Espin

Spain

Khashayar Fakhrian

Germany

Mazda Farshad

Switzerland

Denis Fedorov

Ukraine
Israel Fernandez-Pineda

Spain

Pedro Ferreira

Portugal

Enrico Fiori

Italy

Jochen Franke

Germany

Oren Fruchter

Israel

Sean Galvin

Australia

Shu-Guang Gao

China

Cengiz Gebitekin

Turkey

Ian Geh

United Kingdom

Dechun Geng

China

Pascal Gervaz

Switzerland

Wagih Ghnnam

Egypt

Peter Giannoudis

United Kingdom

Luca Gianotti

Italy

Sonja Gillen

Germany

Montori Giulia

Italy

Gabriel Glockzin

Germany

Stanislaw Gluszek

Poland

Dhanny Gomez

United Kingdom

Jackson A. Gondim

Brazil

Shouping Gong

China

Matthias Goos

Germany
Ajeet Gordhan

USA

Fred Gordon

USA

Rosalie Grivell

Australia

Stephen Grobmyer

USA

Jodok Matthias Grueneberger Germany

Dirk Grünhagen

Netherlands

Salvatore Gruttadauria

Italy

Oleg Gulko

Ukraine

Ali Guner

Turkey

Subhash Gupta

India

Rajesh Gupta

India

Bora Gurer

Turkey

Thilo Hackert

Germany

David Hak

USA

Niels Hammer

Germany

Eric Mark Hammerberg

USA

Jiandong Hao

USA

Jonathan Hardman

United Kingdom

Wolfgang Hartschuh

Germany

Hassan Hassan

USA

Arvid Steinar Haugen

Norway

Alexander Hemprich

Germany 
Ralf Henkelmann

Germany

Lars Henningsohn

Sweden

Benoit Herbert

USA

Noriyuki Hirahara

Japan

Andreas Höch

Germany

Jens Hoeppner

Germany

Werner Hohenberger

Germany

Mathias Hoiczyk

Germany

Peter Hollaus

Germany

Ulrich Theodor Hopt

Germany

Zhiyong Hou

China

Yoshiyuki Hoya

Japan

Karel WE Hulsewe

Netherlands

Igors Iesalnieks

Germany

Abdolmajid Iloon Kashkouli Iran

Daniela Ionescu

Romania

Mitsuru Ishizuka

Japan

Hiroaki Ito

Japan

Kimieru Ito

Japan

Shinji Itoh

Japan

Usman Jaffer

United Kingdom

Sandeep Jain

India
Priyadarshan Jategaonkar

India

Kuo-Shyang Jeng

Taiwan

Jack Jia

China

Jorge Jiménez Cruz

Germany

George Jour

USA

Kethy Jules-elysee

USA

Francis Juthier

France

Daniel Kaemmerer

Germany

Sahin N Kahramanca

Turkey

Adrien Kaladji

France

Erdinc Kamer

Turkey

Kenitiro Kaneko

Japan

Oguz Karahan

Turkey

Cemşit Karakurt

Turkey

Samuel Andreas Käser

Germany

Ozgur Kemik

Turkey

Jeong Han Kim

South Korea

Ji Wan Kim

South Korea

Eugene Kim

USA

Young-Woo Kim

South Korea

Hirokazu Kiyozaki

Japan

Christian Daniel Klink

Germany
Akira Kobayashi

Japan

Kiyotaka Kohshi

Japan

Arto Kokkola

Finland

Eun-jung Kong

South Korea

Manousos Konstadoulakis

Greece

Kostiantyn Kopchak

Ukraine

George Kouvelos

Greece

Bela Kubat

Netherlands

Henry Mark Kuerer

USA

Simon Kuesters

Germany

Vinay Kumaran

India

Anita Kurmann

Switzerland

Miltiadis Lalountas

Greece

Vincent Lam

Australia

Felix Langer

Austria

Hryhoriy Lapshyn

Germany

Bashir Laway

India

Morgan LE Guen

France

Dong Hoon Lee

South Korea

Maw-Sheng Lee

Taiwan

Han Hong Lee

South Korea

Woo Jung Lee

South Korea 
Kuno Lehmann

Switzerland

Ray Leveillee

USA

François L'Hériteau

France

Laura Lorenzon

Italy

Tatiana Lubenets

Ukraine

David Lumenta

Austria

Xiaofeng Lv

China

Vladimir Lyadov

Russian Federation

Giovanni Maconi

Italy

Antonio Macrì

Italy

Almantas Maleckas

Lithuania

Luigi Marano

Italy

Rafik Margaryan

Italy

Goran Marjanovic

Germany

Stephen Mark

New Zealand

Lukas Marti

Switzerland

Shigeki Matsubara

Japan

Ippei Matsumoto

Japan

Blandine Maurel

France

Amine Mazine

Canada

Craig McIlhenny

United Kingdom

Ruth McKee

United Kingdom
Sarah McLaughlin

USA

Ari N. Meguerditchian

Canada

Anastasia Mentessidou

Greece

Aziz Merchant

USA

Juerg Metzger

Switzerland

Yutaka Midorikawa

Japan

Radu Mihai

United Kingdom

Marco Milone

Italy

Aytan Miranda Sipahi

Brazil

Petros Mirilas

USA

Yevgen Miroshnychenko

Ukraine

Suhail Mithani

USA

Teruaki Mizobuchi

Japan

Irwin Mohan

Australia

Yasuhiko Mohri

Japan

Michele Molinari

Canada

Yvonne Mondorf

Germany

Francisco L Moreno Martinez

Cuba

Martin Mortazavi

USA

Ahmet Yaser Muslumanoglu

Turkey

Miki Nagao

Japan

Toru Nagao

Japan
Myura Nagendran

United Kingdom

Takeshi Naitoh

Japan

Yoshifumi Nakao

Japan

Margherita Nannini

Italy

Steven Narod

Canada

Atsushi Nashimoto

Japan

Christian Andreas Nebiker

Switzerland

Valentin Neuhaus

Switzerland

Michael Norwood

United Kingdom

Alexander Novotny

Germany

Dagmar Oberhofer

Croatia

Jooyoung Oh

South Korea

Lawrence Okiror

United Kingdom

Peter O'Leary

Ireland

Terje Osnes

Norway

Hannu Paajanen

Finland

Andrea Pakula

USA

Norbert Pallua

Germany

Stefano Palmucci

Italy

Basilios Papaziogas

Greece

Ji Hoon Park

South Korea

Jeong Yoon Park

South Korea 


\begin{tabular}{|c|c|c|}
\hline $\begin{array}{l}\text { Vani Parmar } \\
\text { India }\end{array}$ & $\begin{array}{l}\text { Asnat Raziel } \\
\text { Israel }\end{array}$ & $\begin{array}{l}\text { Paulus Schurr } \\
\text { Germany }\end{array}$ \\
\hline $\begin{array}{l}\text { Jennifer Paruch } \\
\text { USA }\end{array}$ & $\begin{array}{l}\text { Matthias Reeh } \\
\text { Germany }\end{array}$ & $\begin{array}{l}\text { Andreas Seim } \\
\text { Norway }\end{array}$ \\
\hline $\begin{array}{l}\text { Marta Pascual } \\
\text { Spain }\end{array}$ & $\begin{array}{l}\text { Joachim Reibetanz } \\
\text { Germany }\end{array}$ & $\begin{array}{l}\text { Raffaele Serra } \\
\text { Italy }\end{array}$ \\
\hline $\begin{array}{l}\text { Andrea Patrizi } \\
\text { France }\end{array}$ & $\begin{array}{l}\text { Philipp Renner } \\
\text { Germany }\end{array}$ & $\begin{array}{l}\text { Giuseppe Filiberto Serraino } \\
\text { Italy }\end{array}$ \\
\hline $\begin{array}{l}\text { Milos Pavlovic } \\
\text { Slovenia }\end{array}$ & $\begin{array}{l}\text { Mehmet Reyhan } \\
\text { Turkey }\end{array}$ & $\begin{array}{l}\text { Piergiorgio Settembrini } \\
\text { Italy }\end{array}$ \\
\hline $\begin{array}{l}\text { Florentia Peintinger } \\
\text { Austria }\end{array}$ & $\begin{array}{l}\text { Paul RISS } \\
\text { Austria }\end{array}$ & $\begin{array}{l}\text { Yan-Shen Shan } \\
\text { Taiwan }\end{array}$ \\
\hline $\begin{array}{l}\text { Erman Pektok } \\
\text { Turkey }\end{array}$ & $\begin{array}{l}\text { Andreas J. Roth } \\
\text { Germany }\end{array}$ & $\begin{array}{l}\text { Aali Sheen } \\
\text { United Kingdom }\end{array}$ \\
\hline $\begin{array}{l}\text { Gianluca Pellino } \\
\text { Italy }\end{array}$ & $\begin{array}{l}\text { Andres Mariano Rubiano Escobar } \\
\text { Colombia }\end{array}$ & $\begin{array}{l}\text { Minxin Shi } \\
\text { China }\end{array}$ \\
\hline $\begin{array}{l}\text { Nadia Peparini } \\
\text { Italy }\end{array}$ & $\begin{array}{l}\text { Muhammad Saaiq } \\
\text { Pakistan }\end{array}$ & $\begin{array}{l}\text { Chikashi Shibata } \\
\text { Japan }\end{array}$ \\
\hline $\begin{array}{l}\text { Tirso Perez-Medina } \\
\text { Spain }\end{array}$ & $\begin{array}{l}\text { Anand Sachithanandan } \\
\text { Malaysia }\end{array}$ & $\begin{array}{l}\text { ChungLiang Shih } \\
\text { Taiwan }\end{array}$ \\
\hline $\begin{array}{l}\text { Giuseppe Piccinni } \\
\text { Italy }\end{array}$ & $\begin{array}{l}\text { Babak Saedi } \\
\text { Iran }\end{array}$ & $\begin{array}{l}\text { Satoshi Shiono } \\
\text { Japan }\end{array}$ \\
\hline $\begin{array}{l}\text { Laurentiu Pirtea } \\
\text { Romania }\end{array}$ & $\begin{array}{l}\text { Mauro Salizzoni } \\
\text { Italy }\end{array}$ & $\begin{array}{l}\text { Robert Siegel } \\
\text { Germany }\end{array}$ \\
\hline $\begin{array}{l}\text { John Plukker } \\
\text { Netherlands }\end{array}$ & $\begin{array}{l}\text { Paulina Salminen } \\
\text { Finland }\end{array}$ & $\begin{array}{l}\text { Sumit Sinha } \\
\text { India }\end{array}$ \\
\hline $\begin{array}{l}\text { Elia Poiasina } \\
\text { Italy }\end{array}$ & $\begin{array}{l}\text { Ozgur Samancilar } \\
\text { Turkey }\end{array}$ & $\begin{array}{l}\text { Silvia Siqueira } \\
\text { Brazil }\end{array}$ \\
\hline $\begin{array}{l}\text { Daniel Prevedello } \\
\text { USA }\end{array}$ & $\begin{array}{l}\text { Mohamed Samra } \\
\text { Egypt }\end{array}$ & $\begin{array}{l}\text { Anatolii Skums } \\
\text { Ukraine }\end{array}$ \\
\hline $\begin{array}{l}\text { Pablo Priego } \\
\text { Spain }\end{array}$ & $\begin{array}{l}\text { Andrés Sánchez-Pernaute } \\
\text { Spain }\end{array}$ & $\begin{array}{l}\text { Nicholas Slater } \\
\text { Netherlands }\end{array}$ \\
\hline $\begin{array}{l}\text { Borja Quiroga } \\
\text { Spain }\end{array}$ & $\begin{array}{l}\text { Gabriel Sandblom } \\
\text { Sweden }\end{array}$ & $\begin{array}{l}\text { Matthew Smith } \\
\text { United Kingdom }\end{array}$ \\
\hline $\begin{array}{l}\text { Juan C Quispe } \\
\text { USA }\end{array}$ & $\begin{array}{l}\text { Massimo Sartelli } \\
\text { Italy }\end{array}$ & $\begin{array}{l}\text { Jonathan Sobocinski } \\
\text { France }\end{array}$ \\
\hline $\begin{array}{l}\text { Nikolina Radulovich } \\
\text { Canada }\end{array}$ & $\begin{array}{l}\text { Kirsten Sasaki } \\
\text { USA }\end{array}$ & $\begin{array}{l}\text { Salvatore Sorrenti } \\
\text { Italy }\end{array}$ \\
\hline $\begin{array}{l}\text { Marco Raffaelli } \\
\text { Italy }\end{array}$ & $\begin{array}{l}\text { Atsushi Sasaki } \\
\text { Japan }\end{array}$ & $\begin{array}{l}\text { Michael Sosin } \\
\text { USA }\end{array}$ \\
\hline $\begin{array}{l}\text { Beate Rau } \\
\text { Germany }\end{array}$ & $\begin{array}{l}\text { Jens Schittenhelm } \\
\text { Germany }\end{array}$ & $\begin{array}{l}\text { Cristiano Spadaccio } \\
\text { Belgium }\end{array}$ \\
\hline $\begin{array}{l}\text { Stefano Rausei } \\
\text { Italy }\end{array}$ & $\begin{array}{l}\text { Rick Schneider } \\
\text { Germany }\end{array}$ & $\begin{array}{l}\text { Constantine Spanos } \\
\text { Greece }\end{array}$ \\
\hline
\end{tabular}




Ulrich Spiegl
Germany
Kai Sprengel
Switzerland
Andrew Sprowson
United Kingdom
Michael Stenger
Denmark
Dominika Stygar
Poland
Juan Manuel Suarez Grau
Spain
Hasan Sunar
Turkey
Andrew Swift
United Kingdom
Oleg Symonov
Ukraine
Tomasz Szewczyk
Poland
Yuji Tachimori
Japan
Frank Tacke
Germany
Arash Taheri
USA
Jidenori Takahashi
Jhinsuke Takeno
Jan

Mohammad Talebpour Iran

Dinesh Talwar

United Kingdom

Carol Tan

United Kingdom

Kuniya Tanaka

Japan

Mikhail Tavobilov

Russian Federation

Konstantinos Tepetes Greece

Yuji Toiyama Japan

\begin{tabular}{|c|c|}
\hline $\begin{array}{l}\text { Aybala Tongut } \\
\text { Turkey }\end{array}$ & $\begin{array}{l}\text { Yuedong Wang } \\
\text { China }\end{array}$ \\
\hline $\begin{array}{l}\text { Christos Tourmousoglou } \\
\text { Greece }\end{array}$ & $\begin{array}{l}\text { Atsushi Watanabe } \\
\text { Japan }\end{array}$ \\
\hline $\begin{array}{l}\text { Vincent Traynelis } \\
\text { USA }\end{array}$ & $\begin{array}{l}\text { David Watters } \\
\text { Australia }\end{array}$ \\
\hline $\begin{array}{l}\text { Gary Tse } \\
\text { Hong Kong }\end{array}$ & $\begin{array}{l}\text { Lizzy Weigelt } \\
\text { Switzerland }\end{array}$ \\
\hline $\begin{array}{l}\text { Akif Turna } \\
\text { Turkey }\end{array}$ & $\begin{array}{l}\text { Ulrich Friedrich Wellner } \\
\text { Germany }\end{array}$ \\
\hline $\begin{array}{l}\text { Garth Utter } \\
\text { USA }\end{array}$ & $\begin{array}{l}\text { Sandy Widder } \\
\text { Canada }\end{array}$ \\
\hline $\begin{array}{l}\text { Todd Vander Heiden } \\
\text { USA }\end{array}$ & $\begin{array}{l}\text { Philipp Wiggermann } \\
\text { Germany }\end{array}$ \\
\hline $\begin{array}{l}\text { Horatiu Vasian } \\
\text { Romania }\end{array}$ & $\begin{array}{l}\text { Allison Williams } \\
\text { USA }\end{array}$ \\
\hline $\begin{array}{l}\text { Mohammad Vaziri } \\
\text { Iran }\end{array}$ & $\begin{array}{l}\text { Donna Williams } \\
\text { USA }\end{array}$ \\
\hline $\begin{array}{l}\text { Ali Veral } \\
\text { Turkey }\end{array}$ & $\begin{array}{l}\text { Uwe Wittel } \\
\text { Germany }\end{array}$ \\
\hline $\begin{array}{l}\text { Andras Vereczkei } \\
\text { Hungary }\end{array}$ & $\begin{array}{l}\text { Reuben Wong } \\
\text { Singapore }\end{array}$ \\
\hline $\begin{array}{l}\text { Jorrit Jan Verlaan } \\
\text { Netherlands }\end{array}$ & $\begin{array}{l}\text { Chaoneng Wu } \\
\text { China }\end{array}$ \\
\hline $\begin{array}{l}\text { Nereo Vettoretto } \\
\text { Italy }\end{array}$ & $\begin{array}{l}\text { Jianwei Xie } \\
\text { China }\end{array}$ \\
\hline $\begin{array}{l}\text { Ishwarappa Balekundri Vijayalakshmi } \\
\text { India }\end{array}$ & $\begin{array}{l}\text { Katsuhiko Yanaga } \\
\text { Japan }\end{array}$ \\
\hline $\begin{array}{l}\text { Charles Vincent } \\
\text { United Kingdom }\end{array}$ & $\begin{array}{l}\text { Hee Chul Yang } \\
\text { South Korea }\end{array}$ \\
\hline $\begin{array}{l}\text { Minutolo Vincenzo } \\
\text { Italy }\end{array}$ & $\begin{array}{l}\text { Chun-Nan Yeh } \\
\text { Taiwan }\end{array}$ \\
\hline $\begin{array}{l}\text { Brendan Visser } \\
\text { USA }\end{array}$ & $\begin{array}{l}\text { Erdal Yekeler } \\
\text { Turkey }\end{array}$ \\
\hline $\begin{array}{l}\text { Ernst von Dobschuetz } \\
\text { Germany }\end{array}$ & $\begin{array}{l}\text { Christopher Young } \\
\text { Australia }\end{array}$ \\
\hline $\begin{array}{l}\text { Rüdiger M O Von Eisenhart-Rothe } \\
\text { Germany }\end{array}$ & $\begin{array}{l}\text { Takafumi Yukaya } \\
\text { Japan }\end{array}$ \\
\hline $\begin{array}{l}\text { Urs Von Holzen } \\
\text { Switzerland }\end{array}$ & $\begin{array}{l}\text { Giovanni Zagli } \\
\text { Italy }\end{array}$ \\
\hline $\begin{array}{l}\text { Nicolas H. von der Hoeh } \\
\text { Germany }\end{array}$ & $\begin{array}{l}\text { Dirk Zajonz } \\
\text { Germany }\end{array}$ \\
\hline $\begin{array}{l}\text { Michael Wall } \\
\text { Australia }\end{array}$ & $\begin{array}{l}\text { Denise Zantut Wittmann } \\
\text { Brazil }\end{array}$ \\
\hline
\end{tabular}


Kamran Zargar Shoshtari

New Zealand

Sergii Zemskov

Ukraine

Enver Zerem

Bosnia and Herzegovina
Yingze Zhang

China

Chunfeng Zhao

USA

Jiawei Zheng

China
Cheng Zhou

China

Xuesong Zhu

China

Olexiy Zubkov

Ukraine

Received: 20 January 2015 Accepted: 20 January 2015 Published: 13 February 2015

doi:10.1186/1471-2482-15-8

Cite this article as: Tu: BMC Surgery reviewer

acknowledgement, 2014. BMC Surgery 2015 15:8. 